\title{
A avaliação do craving em alcoolistas na síndrome de abstinência
}

\author{
Renata Brasil Araújo ${ }^{1}$ \\ Margareth da Silva Oliveira \\ Maria Lúcia Tiellet Nunes \\ Luciane Benvegnu Piccoloto \\ Wilson Vieira Melo
}

\begin{abstract}
Resumo
O craving pode ser entendido como um desejo de utilizar uma substância. Este estudo pretende verificar características do craving em alcoolistas, nos primeiros três dias de abstinência, comparar sua intensidade com a do período de consumo do álcool e verificar se está associado com a gravidade do alcoolismo. A amostra foi de 77 homens alcoolistas, internados para desintoxicação. Foi utilizada a Escala de Avaliação do Craving e o Short-Form Alcohol Dependence Data. Destes sujeitos, 58,2\% nunca sentiu vontade de utilizar bebidas alcoólicas nos três primeiros dias de abstinência. Quanto ao período antes da internação, 90,9\% relatou apresentar craving, mais intenso durante essa fase de consumo do que na abstinência. Obteve-se correlação positiva entre o craving e a gravidade da dependência. Conhecer as características do craving de alcoolistas torna possível ampliar as técnicas de prevenção de recaída.

Palavras-Chave: Álcool; Craving; Desintoxicação.
\end{abstract}

\section{The craving evaluation in alcoholics in abstinence syndrome}

\begin{abstract}
Craving can be understood as an intense desire for substance consumption. This study intends to check craving characteristics in alcoholics during the first three days of abstinence syndrome, compare its intensity to the one during the alcohol consumption period, and verify if craving is associated with the degree of alcohol dependence. The sample is formed by 77 male alcoholics interned for detoxication. Scale for Craving Evaluation and a Short-Form Alcohol Dependence Data were applied. $58.2 \%$ of the participants never will to drink alcoholic beverages on the first three days of abstinence. Concerning the time before hospitalization, 90.9\% stated feeling craving and during consumption stage it was more intense than during abstinence. There was positive correlation between craving and degree of alcohol dependence. Knowing about the craving characteristics in alcoholics makes possible to enhance techniques of relapse prevention.
\end{abstract}

Keywords: Alcohol; Craving; Detoxication.

A dependência de uma substância química como o álcool é uma um diagnóstico freqüentemente encontrado na clínica, sendo fundamental a compreensão de suas implicações para que se possa lidar, de uma forma mais efetiva, com este problema (Edwards, Marshall \& Cook, 1999). A desintoxicação do álcool, é importante destacar, é uma das mais difíceis, em virtude dos sintomas físicos da sua característica síndrome de abstinência, que inclui sudorese, hipertensão arterial, taquicardia, tremores, convulsões, podendo, até mesmo, ocorrer um quadro de Delirium Tremens, quando são acrescidos delírios e alucinações. Um adequado monitoramento do paciente nesta fase deve ser uma das preocupações do profissional que atende a esta clientela (Ramos \& Galperim, 1997).

A síndrome de abstinência do álcool geralmente é superada até o final dos primeiros sete dias de tratamento, sendo uma oportunidade para fortalecer o vínculo terapêutico e para que o paciente possa dar-se conta dos prejuízos causados por sua dependência (Ramos \& Galperim, 1997). De acordo com Beck, Wright, Newman e Liese (1999), o craving ou "fissura" (desejo intenso de usar a substância) é um fator que pode estar presente tanto durante o uso da substância como na fase de desintoxicação ou, ainda, após uma interrupção mais prolongada, sendo este, segundo Hongstrom, Thorburn, Hiltunen e Borg (1999), um dos sinais de alerta que poderia levar a um lapso ou recaída.

Segundo Marlatt e Gordon (1993), o craving pode ser definido como um estado motivacional subjetivo influenciado pelas expectativas associadas a um resultado positivo; tal estado pode induzir uma resposta na qual o comportamento desejado esteja envolvido. Este desejo de difícil controle - o craving - pode ser desencadeado por sugestões cognitivas internas ou ambientais, sendo um dos principais objetivos esperados do paciente descobrir como este "gatilho" pode

${ }^{1}$ Endereço para correspondência:

Av. Ipiranga, 6.681, Prédio 11 - 9o andar, sala 932 - Porto Alegre-RS - 90619-900

E-mail: renataudbrasil@hotmail.com 
desencadear uma situação de risco para uma recaída (Edwards \& Dare, 1997).

O craving, de acordo com Marlatt e Gordon (1993), pode ser considerado uma variável interveniente; interpõe-se entre uma causa (por exemplo, a síndrome de abstinência do álcool, ou uma situação estressante) e uma resposta (neste caso, o ato de beber). Caso esse conceito não seja operacionalmente definido, sendo esclarecidas as variáveis dependentes e independentes associadas, pode ser considerado a causa do comportamento manifesto, o que ocorre em alguns modelos, como o "Modelo Doença", que acredita que ele é um sintoma decorrente de uma necessidade interna fisiológica por uma substância que causa a perda do controle do ato de consumir este produto. Cabe distinguir craving de compulsão, sendo a segunda um comportamento decorrente da vontade subjetiva causada pelo primeiro. Beck et al. (1999) referem que o objetivo do impulso é reduzir o craving e buscar um estado de relaxamento. É interessante chamar a atenção para o fato de que, freqüentemente, esses dois termos são utilizados indiscriminadamente. De acordo com alguns autores (Cox, Tiffany \& Christen, 2001; Tiffany \& Drobes, 1991), por outro lado, o construto craving é mais abrangente e está relacionado a quatro categorias: desejo de fumar; antecipação de resultado positivo; alívio dos sintomas de privação ou efeito negativo e intenção de fumar, sendo estas categorias o que impulsiona o indivíduo a ter um comportamento de busca pela droga.

Ainda que o craving, que leva uma pessoa a consumir o álcool, seja incontrolável, é possível que o impulso despertado seja dominado voluntariamente, mesmo que o indivíduo sinta-se incapaz para tal. Este desejo de evitar o consumo tem um componente cognitivo importante: a tomada de decisões. Esse autocontrole não se expressa de uma forma visceral como o craving, sendo estas motivações opostas e qualitativamente distintas (Beck et al., 1999). Independentemente das questões conceituais, é importante definir quais foram as situações que o estimularam e os impulsos relacionados ao uso da bebida alcoólica, para que sejam mais bem trabalhadas as estratégias de prevenção de recaída (Marlatt \& Gordon, 1993).

Foram realizados estudos que concluíram que imagens mentais associadas ao álcool interferem no seu aumento e a disponibilização deste conhecimento e seu potencial para desencadear uma recaída pode ajudar o paciente em termos de auto-eficácia, pois o instrumentaliza a saber que determinadas situações ou cenas devem ser evitadas para não comprometer sua abstinência (Araújo, 2002; Marlatt \& Gordon, 1993; Weinstein, Lingford, Martinez \& Marshall, 1998). O craving tem sido relacionado, por alguns autores, às particularidades de cada substância psicotrópica utilizada conjuntamente com as características individuais dos dependentes químicos, não podendo ser entendido sem que a interrelação destes fatores seja contemplada (Marques \& Seibel, 2001; Singleton \& Gorelick, 1998).

Em razão da importância do craving no tratamento de comportamentos dependentes, alguns autores preocuparam-se em estudar mais a respeito deste tema e, inclusive, desenvolver instrumentos para a sua mensuração (Dols, Hout, Kindt \& Willems, 2000a; Dols, Willems, Hout \& Bittoun, 2000b; Miyata \& Yanagita, 2001; Sweeney, Pillitteri \& Kozlowski, 1996; Tiffany \& Drobes, 1991). Este artigo tem por objetivo identificar, por meio de uma escala para avaliar o craving (Araújo, 2002), suas características em pacientes alcoolistas nos primeiros dias de desintoxicação, bem como comparálo com o craving avaliado durante a fase de consumo do álcool. Também pretende avaliar se há correlação entre o craving e a gravidade da dependência dessa substância.

\section{Método}

\section{Participantes}

Este foi um estudo transversal e correlacional, no qual foi utilizada uma amostra "por conveniência", ou "não-aleatória", de 77 sujeitos, dependentes do álcool, do sexo masculino, com escolaridade mínima

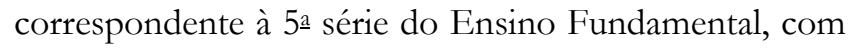
um faixa etária entre de 18 e 65 anos, internados em Unidades de Desintoxicação. O último dia de ingesta de bebida alcoólica deveria ser nas 24 horas precedentes à internação e os pacientes não poderiam estar utilizando nenhuma medicação psiquiátrica, exceto benzodiazepínico e tiamina. Foram excluídos aqueles pacientes que fizeram menos de 25 pontos no Miniexame do Estado Mental, que tinham co-morbidades psiquiátricas incapacitantes como Esquizofrenia, Transtornos do Humor, Demência, Síndrome Amnéstica, Síndrome Cerebral Orgânica, ou que apresentaram sintomas de abstinência que alterassem o desempenho nos testes e que dependessem de outras substâncias psicoativas, salvo a nicotina.

\section{Instrumento}

O instrumento aplicado foi uma ficha com dados sociodemográficos com o objetivo de definir o perfil da amostra estudada, identificando características que eram importantes para melhor avaliar se o indivíduo podia ou não ser incluído na amostra. Esta era preenchida durante uma entrevista para coleta de dados, sendo levantadas hipóteses diagnósticas de acordo com a CID-10 (Organização Mundial da Saúde, 1993), realizado o exame do estado mental de cada paciente (Kaplan, Sadock \& Grebb, 1997) e avaliada a quantidade de álcool consumida diariamente. Em virtude de ser dificultada a mensuração do consumo do 
álcool por existirem diferenças na quantidade de suas doses e variações na sua concentração, de acordo com cada tipo de bebida e com os diferentes países nos quais esta substância é produzida, optou-se, pela utilização da medida diária "Unidades Internacionais de Álcool" (UI), as quais são calculadas dividindo-se a quantidade de álcool consumida (em mililitros) por 30, logo após, multiplicando esse resultado pela concentração de álcool da bebida utilizada e, este total, por 2 (Oliveira 2000). Foi utilizado, nesta pesquisa, para excluir da amostra os pacientes com prejuízo cognitivo, o Miniexame do Estado Mental - Mini-Mental State Examination: MMSE (Folstein \& McHugh, 1975; Kaplan et al., 1997), que é um teste de triagem muito útil em exame clínico de um paciente. Tem como objetivo avaliar o estado cognitivo, sendo que uma pontuação de 25 do total de 30 pontos sugere comprometimento e abaixo de 20 pontos indica, com certeza, que há um prejuízo cognitivo.

Outro instrumento utilizado foi o questionário Short-Form Alcohol Dependence Data - SADD, criado por Raistrick em 1983 e padronizado para uso no Brasil por Jorge e Mansur (1986), para avaliar o grau de gravidade da dependência do álcool. É constituído de 15 questões, com 4 opções de respostas $(0$ - nunca, 1 - poucas vezes, 2 - muitas vezes e 3 -sempre). Classifica a dependência em: leve (0-9), moderada (10-19) e grave (20-45). Este questionário foi utilizado para avaliar a gravidade da dependência do álcool da amostra pesquisada, o que auxiliou na identificação do perfil da mesma.

$\mathrm{O}$ último instrumento do protocolo deste estudo foi a Escala para Avaliar o Craving (Araújo, 2002), construída e testada em um estudo piloto, tendo este processo de construção e validação inicial se fundamentado na literatura relacionada a questionários de avaliação deste construto (Anton, Moak \& Latham, 1995; Beck et al., 1999; Flannery, Volpicelli \& Pettinati, 1999; Marques \& Seibel, 2001). A produção preliminar foi avaliada por profissionais especialistas em dependência química e, após as correções, o instrumento foi aplicado em dez pacientes. O objetivo dessa aplicação "piloto" foi verificar se o instrumento estava adequado tanto gramatical quanto funcionalmente. Foi realizado um estudo estatístico dessa escala, obtendo-se um alfa de Cronbach igual a 0,89 , o que comprovou a fidedignidade da mesma. A Escala para Avaliação do Craving é constituída por 11 questões objetivas que se referem ao craving avaliado nos três primeiros dias de internação. A intensidade do craving foi mensurada com base no somatório dos pontos e a classificação feita em quatro níveis: insignificante, de 0-5 pontos; leve, de 6-14 pontos; média, de $15-25$ pontos; e forte, acima de 26 pontos. A escala foi composta por questões referentes ao craving percebido nos últimos três dias de internação, havendo uma questão relacionada a três dias antes do indivíduo internar, quando estava consumindo a bebida alcoólica.

\section{Procedimentos}

Primeiramente, o projeto desta pesquisa foi avaliado pelos Comitês de Ética dos hospitais onde ela foi realizada e, a partir da aprovação destas instâncias, iniciou-se a coleta de dados propriamente dita. Antes de cada indivíduo aceitar fazer parte da pesquisa, mesmo durante o estudo piloto, era explicada a finalidade da pesquisa e que ela era regida por princípios éticos, sendo fornecido o Termo de Consentimento Livre e Esclarecido. A assinatura deste Termo era uma précondição para que o sujeito fosse incluído na amostra.

Cada paciente com o perfil da amostra foi encaminhado, no quarto dia de abstinência, individualmente, a uma sala onde era feita uma entrevista de avaliação, preenchida uma Ficha com Dados Sociodemográficos e aplicado o Miniexame do Estado Mental. Os pacientes que não preencheram nenhum critério de exclusão foram finalmente incluídos na amostra, e a estes foi mensurado o craving pela escala criada para tal (Araújo, 2002).

\section{Análise de Dados}

Os estudos estatísticos foram os descritivos para analisar a freqüência do perfil sociodemográfico da amostra, bem como as características do craving. Também, a prova do qui-quadrado para avaliar se havia uma diferença significativa entre o craving na fase de desintoxicação e de consumo do álcool e o coeficiente de correlação de Spearman para analisar a correlação entre o craving e a gravidade da dependência do álcool.

\section{Resultados}

A amostra foi constituída por 77 sujeitos, do sexo masculino, internados em duas unidades especializadas em dependência química, 88,3\% na instituição pública e $11,7 \%$ na privada. A idade média dos sujeitos foi de 41,53 anos (desvio padrão=8,825), com limites inferior e o superior equivalentes a 18 e 63 anos, respectivamente.

A média de idade com a qual os indivíduos experimentaram a bebida alcoólica foi de 18,94 anos (desvio padrão=6,7), com os limiares inferior e superior correspondentes, na ordem, a 8 e 40 anos. O número total de internações na vida de cada sujeito pesquisado teve média igual a 3,94 (desvio padrão=5,34). A quantidade média de álcool consumida diariamente, antes da internação, foi de 31,71 UI (Unidades Internacionais) com desvio padrão $=22,58(n=77)$. Quanto à gravidade da dependência, 1,4\% apresentava nível leve $(n=8) ; 32,5 \%(n=25)$, moderado e $57,1 \%(n=44)$, grave.

Ao analisar a pontuação total da escala para avaliar o craving, a média foi de 12,22 pontos (desvio 
padrão=10,55), sendo o escore mínimo 0 (zero) e o

craving estão mais bem descritas na Tabela 1.

máximo 40 pontos. As pontuações relacionadas ao

Tabela 1 - Análise descritiva do craving

\begin{tabular}{|c|c|c|}
\hline Variáveis & $\mathrm{N}$ & $\%$ \\
\hline \multicolumn{3}{|l|}{ Pontuação do craving durante a internação } \\
\hline Nota zero - não teve fissura & 45 & 58,4 \\
\hline Entre 1 e 2 - fissura fraca & 18 & 23,4 \\
\hline Entre 3 e 4 - fissura média/fraca & 6 & 7,8 \\
\hline Entre 5 e 6 - fissura média/fraca & 2 & 2,6 \\
\hline Entre 7 e 8 - fissura forte & 4 & 5,2 \\
\hline Entre 9 e 10 - fissura muito forte & 2 & 2,6 \\
\hline \multicolumn{3}{|l|}{ Freqüência em que ocorreu o craving } \\
\hline Em nenhum dia & 45 & 58,4 \\
\hline Às vezes & 19 & 34,5 \\
\hline Muitas vezes & 2 & 3,6 \\
\hline Sempre & 2 & 3,6 \\
\hline \multicolumn{3}{|l|}{ Tempo de duração do craving } \\
\hline Não teve & 45 & 58,4 \\
\hline Minutos & 17 & 30,9 \\
\hline De 1 a 2 horas & 3 & 5,5 \\
\hline De 3 a 4 horas & 1 & 1,8 \\
\hline Mais de 4 horas & 2 & 3,6 \\
\hline \multicolumn{3}{|c|}{ Distribuição do craving por dia de internação } \\
\hline Não teve fissura & 45 & 58,4 \\
\hline No 1익 & 8 & 21,8 \\
\hline No 2o dia & 5 & 9,1 \\
\hline No 3o dia & 4 & 7,6 \\
\hline Em mais de um dia & 2 & 3,6 \\
\hline \multicolumn{3}{|l|}{ Turno em que o craving era mais intenso } \\
\hline Não teve fissura & 45 & 58,4 \\
\hline Manhã & 6 & 10,9 \\
\hline Tarde & 4 & 7,3 \\
\hline Noite & 8 & 14,5 \\
\hline Variava & 2 & 3,1 \\
\hline Em vários turnos & 3 & 5,5 \\
\hline \multicolumn{3}{|c|}{ Craving 3 dias antes da internação - fase de consumo } \\
\hline Nota zero - não teve fissura & 8 & 10,4 \\
\hline Entre 1 e 2 - fissura fraca & 6 & 7,8 \\
\hline Entre 3 e 4 - fissura média/ fraca & 11 & 14,3 \\
\hline Entre 5 e 6 - fissura média/ fraca & 11 & 14,3 \\
\hline Entre 7 e 8 - fissura forte & 13 & 16,9 \\
\hline Entre 9 e 10 - fissura muito forte & 28 & 36,4 \\
\hline Total & 77 & 100 \\
\hline
\end{tabular}

A análise estatística dos dados permite afirmar a existência de diferença significativa entre o craving na fase de consumo e durante a internação $\left(X^{2}=14,18\right.$; $\mathrm{p}=0,000$ ), sendo constatado que o craving durante a fase de consumo é mais intenso. Além disso, foi encontrada uma correlação significativa, positiva e de intensidade média entre o craving e a gravidade da dependência do álcool medida pelo SADD $(\underline{\underline{r}} s=0,509 ; \mathrm{p}=0,000)$.

\section{Discussão dos Resultados}

Vários autores (Beck et al., 1999; Edwards et al., 1999; Marlatt \& Gordon, 1993; Ramos \& Galperim, 1997) descrevem como o tratamento de dependentes de álcool requer cuidados, pois, tanto do ponto de vista clínico como psicológico, existem várias questões implicadas, como, por exemplo, a característica síndrome de 
abstinência, os danos clínicos e neurológicos e o desejo intenso de consumir a substância (craving) que, muitas vezes, insiste em acompanhar o indivíduo, mesmo após um longo tempo em abstinência (Beck et al., 1999; Edwards \& Dare, 1997; Hongstrom et al., 1999; Marlatt \& Gordon, 1993; Weinstein et al., 1998). Como comportamentos dependentes são multifatoriais (Ramos \& Galperim, 1997), qualquer análise deve contemplar tantos aspectos quanto possíveis pelo delineamento das pesquisas, de modo a não partir para conclusões criticáveis por tratar o problema de forma isolada ou ingênua.

Um ponto a destacar refere-se ao fato de que mais da metade da amostra afirmou não ter sentido vontade de beber durante a internação, pois 58,4\% atribuiu a nota zero à média do craving nestes dias; a pontuação média, nesta escala específica, foi de 12,22 pontos (desvio padrão=10,55), o que é classificado por esta escala como "fraco" (Araújo, 2002). Estes dados se fazem acompanhar de outra informação, qual seja, apenas $6,8 \%$ dos sujeitos tiveram um desejo muito intenso de beber na fase inicial da desintoxicação. Nesse caso, o fato de os pacientes estarem internados em um ambiente protegido de estímulos envolvendo o álcool pode explicar a atenuação do desejo de beber nesta amostra, o que estaria de acordo com estudos de Edwards e Dare (1997) e Weinstein et al. (1998), pelos quais imagens mentais ligadas à bebida alcoólica e sugestões cognitivas internas ou ambientais interferem no aumento do craving, sendo importante a evitação das mesmas para o controle do craving e para a manutenção da abstinência.

Esses resultados se evidenciam também via aqueles obtidos pela medida aumentada do craving na fase de consumo de álcool, quando os sujeitos se reportaram à situação em que ainda estavam diante de vários estímulos agregados à bebida alcoólica. A pontuação na escala de craving foi mais alta nesta fase de consumo do que na de abstinência, sendo encontrada uma diferença significativa do ponto de vista estatístico.

Pode-se afirmar que, por ser mais intenso durante a fase de consumo, é necessário o ensino de estratégias de seu manejo para todos os pacientes na clínica, desde o início do tratamento, de modo que possam iniciar a interrupção do uso da bebida alcoólica, estando mais instrumentalizados para tal. Os resultados também apontam para a necessidade de intervenções que visem à prevenção primária e secundária, à medida que os pacientes mais graves são os que relatam mais intenso craving e quanto mais cedo seja feita uma abordagem, mais condições estes indivíduos terão para controlar o seu desejo pelo álcool.

Foi possível evidenciar que pacientes alcoolistas internados para desintoxicação, impedidos de beber não só por questões referentes à vontade própria, mas por controle ambiental que impossibilitava o acesso a bebidas alcoólicas, não apresentaram uma intensa vontade de beber. Portanto, a internação hospitalar pode ser um dos tratamentos de escolha para desintoxicação por oferecer um local de continência e controle para este desejo tão difícil de ser aplacado, como é o de beber. Em alguns casos, este desejo é tão intenso que torna contra-indicado o tratamento destes indivíduos $\mathrm{em}$ regime ambulatorial. É necessário, entretanto, considerar o fato de que alguns pacientes querem demonstrar uma melhora em seu quadro para, conseqüentemente, antecipar a sua alta, isso podendo vir a ser um viés quando se analisa a variável craving durante uma internação hospitalar.

O tema craving é comum de ser escutado na clínica do dependente químico. $\mathrm{Na}$ verdade, os profissionais e os pacientes o percebem como um elemento que pode aparecer a qualquer momento, mesmo após um longo tempo em abstinência e que, vencendo a habilidade de enfrentamento do indivíduo, é capaz de desencadear uma recaída. O problema é que, muitas vezes, ele aparece sem aviso prévio, ou os terapeutas, juntamente com os pacientes, não conseguem detectá-lo a tempo, o que dificulta o seu manejo e diminui a autoeficácia do sujeito que, com menos estratégias de enfrentamento, termina por ter um lapso ou uma recaída.

Por isso, a utilização de instrumentos para avaliar o craving pode ser importante para detectar sua presença antes que seja iniciada uma interrupção do consumo da bebida alcoólica, durante o processo de desintoxicação e mesmo após um período maior de abstinência, sendo um auxílio para os profissionais e para os pacientes durante todo o processo terapêutico.

\section{Referências}

Anton, R. F., Moak, D. H. \& Latham, P. (1995). The Obsessive Compulsive Drinking Scale: a self-rated instrument for the quantification of thoughts about alcohol and drinking behavior. Alcoholism: Clinical and Experimental Research, 19, 1, 92-99.

Araújo, R. B. (2002). A relação entre sonhos e craving em alcoolistas na fase de desintoxicação. (Dissertação de Mestrado). Porto Alegre: PUCRS.

Beck, Aaron T., Wright, Fred D., Newman, Corey F. \& Liese, Bruce S. (1999). Terapia cognitiva de las drogodependencias. Barcelona: Paidós.

Cox, L. S., Tiffany, S. T. \& Christen, A. G. (2001). Evaluation of the brief questionnaire of smoking urges (QSU-brief) in laboratory and clinical settings. Nicotine \& Tobacco Research, 3, 7-16.

Dols, M., Hout, Van den, Kindt, M \& Willems, B. (2000a). The urge to smoke depends on the expectation of smoking. Addiction, 97, 87-93.

Dols, M., Willems, M., Hout, Van den \& Bittoun, R. 
(2000b). Smokers can learn to influence their urge to smoke. Addictive Behaviors, 25(1), 103-108.

Edwards, G. \& Dare, C. (1997). Psicoterapia e tratamento das adicõos. Porto Alegre: Artes Médicas.

Edwards, G., Marshall, E. Jane \& Cook, C. C. H. (1999). $O$ tratamento do alcoolismo. Porto Alegre: Artes Médicas.

Flannery, B. A., Volpicelli, J. R. \& Pettinati, H. M. (1999). Psychometric properties of the Penn Alcohol Craving Scale. Alcoholism: Clinical and Experimental Research, 23, 1289-1295.

Folstein, M. \& McHugh, P. R. (1975). Mini-mental state: A practical method for grading the cognitive state of patient for the clinician. Journal of Psychiatry Research, 12, 189.

Hongstrom, B. A., Thorburn, D., Hiltunen, A. \& Borg, S. (1999). Prediction of single episodes of drinking during the treatment of alcohol-dependent patients. Sweden. Alcohol, 18(1), 35-42.

Jorge, M. R. \& Mansur, J. (1986). Questionários padronizados para Avaliação do grau de Severidade da Síndrome de Dependência do Álcool. Jornal Brasileiro de Psiquiatria, 35(5), 287-292.

Kaplan, H., Sadock, B. \& Grebb, J. A. (1997). Compêndio de Psiquiatria - Ciências do Comportamento e Psiquiatria Clínica. Porto Alegre: Artes Médicas.

Marlatt, A. \& Gordon, J. (1993). Prevenção de recaída estratégias de manutenção no tratamento de comportamentos adictivos. Porto Alegre: Artes Médicas.

Marques, A. C. \& Seibel, S. D. (2001). O craving. Em Seibel, S. D. \& Toscano Jr. Dependência de drogas. (pp. 239-248). São Paulo: Atheneu.

Miyata, H. \& Yanagita, T. (2001). Neurobiological mechanism of nicotine craving. Alcohol, 24, 87-93.
Oliveira, M. (2000). Eficácia da intervenção motivacional em dependentes do álcool. (Dissertação de Doutorado). São Paulo: Universidade Federal de São Paulo.

Organização Mundial de Saúde. (1993). Classificação de transtornos mentais e de comportamento da CID-10: descriçooes clínicas e diretrizes diagnósticas. Porto Alegre: Artes Médicas.

Raistrick, D., Dunbar, G. \& Robinson, D. (1983). Development of a questionnaire to measure alcohol dependence. British Journal of Addiction, 78, 89-95.

Ramos, S. \& Galperim, B. (1997). Desintoxicação. Em S. Ramos \& J. M. Bortolote. Alcoolismo hoje. (pp. 149159). Porto Alegre: Artes Médicas.

Singleton, E. G. \& Gorelick, D. A. (1998). Mechanism of alcohol craving their clinical implications. Em Galanter, M. (Org.). Recent developments in alcoholism. vol. 14. (pp. 177-195). The Consequences of Alcoholism. New York: Plenum Press.

Sweeney, C., Pillitteri, J. \& Kozlowski, L. T. (1996). Measuring drug urges by questionnaire: do not balance scales. Addictive Behaviors, 21 (2) 199-204.

Tiffany, S. T. \& Drobes, D. J. (1991). The development and initial validation of a questionnaire on smoking urges. British Journal of Addiction, 86, 1467-1476.

Weinstein, A., Lingford, Hughes A., Martinez, Raga J. \& Marshall, J. (1998). What makes alcohol-dependent individuals early in abstinence crave for alcohol: exposure to the drink, images of drinking, or remembrance of drinks past? Alcoholism: Clinical and Experiemental Research, 22(6), 1376-1381.

Enviado em março de 2004 Reformulado em maio de 2004 Aprovado em maio de 2004

Sobre os autores:

Renata Brasil Araújo é psicóloga, professora da residência do Hospital Psiquiátrico São Pedro, doutoranda em Psicologia, coordenadora da residência integrada em Saúde Mental do Hospital Psiquiátrico São Pedro.

Margareth da Silva Oliveira é psicóloga, doutora em Ciências, professora titular na Faculdade de Psicologia da PUCRS, atuando nos cursos de graduação e pós-graduação, e coordenadora do Grupo de Pesquisa Intervenções Cognitivas e Comportamentos Dependentes.

Maria Lucia Tiellet Nunes é psicóloga, doutora em Psicologia pela Universidade Livre de Berlim, professora titular na Faculdade de Psicologia da PUCRS, atuando nos cursos de graduação e pós-graduação.

Luciane Benvegnu Piccoloto é psicóloga clínica, mestre em Psicologia Clínica, professora da Universidade Luterana do Brasil e professora e membro do corpo clínico da WP - Centro de Psicoterapia Cognitivo Comportamental.

Wilson Vieira Melo é psicólogo clínico, mestrando em Psicologia Clínica, professor e membro do corpo clínico da WP - Centro de Psicoterapia Cognitivo Comportamental. 